\title{
Acknowledgement to reviewers of Cancer Drug Resistance in 2019
}

\author{
Cancer Drug Resistance Editorial Office \\ OAE Publishing Inc., Chaoyang District, Beijing 100016, China.
}

Correspondence to: OAE Publishing Inc., Rm. 968, 9th Floor, Xinhengji International Building, No. 3 Maizidian West Road, Chaoyang District, Beijing 100016, China.E-mail: editorial@cdrjournal.com

How to cite this article: Cancer Drug Resistance Editorial Office. Acknowledgement to reviewers of Cancer Drug Resistance in 2019. Cancer Drug Resist 2020;3:2020;3:110-2. http://dx.doi.org/10.20517/cdr.2020.02

Received: 6 Jan 2020 Accepted: 7 Jan 2020 Published: 19 Mar 2020

Science Editor: Frits Peters Copy Editor: Jing-Wen Zhang Production Editor: Jing Yu

The editors of the journal Cancer Drug Resistance (CDR) would like to take this opportunity to express their sincere gratitude to the following reviewers for assessing the manuscripts in 2019 [Table 1].

Table 1. The reviewers in 2019

\begin{tabular}{lll}
\hline Names & & \\
\hline Abdelmohsen, Kotb & Holler, Eggehard & Puri, Sonam \\
Abe, Tatsuya & Hydbring, Per & Ricciardelli, Carmela \\
Altucci, Lucia & Jacinto, Estela & Rigaud, Michel \\
Amantini, Consuelo & Jansen, Gerrit & Ritter, Christoph \\
Asao, Tetsuhiko & Jenkins, Frank J. & Robert, Jacques \\
Azmi, Asfar & Jiménez-Morales, Silvia & Roberts, Arthur \\
Baba, Tomohisa & Jogalekar, Manasi & Rocca, Andrea \\
Ballinger, James R. & Johns, Terrance & Rodolfo, Monica \\
Beck, William T. & Jolly, Mohit Kumar & Rodrigues, Antonio Sebastião \\
Beckermann, Kathryn Eby & Ju, Jingfang & Rodriguez, Jose Antonio \\
Bednarski, Patrick J. & Kalayda, Ganna & Rodríguez-Antona, Cristina \\
Benny, Ofra & Kanda, Tatsuo & Rossana, Berardi \\
Bessho, Tadayoshi & Kannan, Pavitra & Rueff, José \\
Boland, Genevieve & Kaoud, Tamer & Rupasinghe, H.P. Vasantha \\
Bottone, Maria Grazia & Ketter, Ralf & Saffi, Jenifer \\
Bourdon, Jean-Christophe & Keyse, Steven & Sato, Akinori \\
Branco, Pedro Castelo & Knudsen, Birgitta & Scarpi, Emanuela \\
Buchbinder, Elizabeth I. & Koczor, Chris & Schwarzenbach, Heidi \\
Buchler, Tomas & Krajinović, Maja & Shah, Khyati
\end{tabular}

cC) (7) $\odot$ The Author(s) 2020. Open Access This article is licensed under a Creative Commons Attribution 4.0 International License (https://creativecommons.org/licenses/by/4.0/), which permits unrestricted use, sharing, adaptation, distribution and reproduction in any medium or format, for any purpose, even commercially, as long as you give appropriate credit to the original author(s) and the source, provide a link to the Creative Commons license, and indicate if changes were made. 


\begin{tabular}{|c|c|c|}
\hline Budunova, Irina & Kwok, Hang Fai & Shimono, Yohei \\
\hline Calvert, Hilary & Lahn, Michael M & Simanshu, Dhirendra K. \\
\hline Cappelletti, Vera & Lauschke, Volker M & Sionov, Ronit Vogt \\
\hline Carnero, Amancio & Lavrik, Olga & Skaar, Todd C. \\
\hline Cascorbi, Ingolf & Lee, Yuan-Hao (Chris) & Somasagara, Ranga \\
\hline Cecchin, Erika & Leo, Eliana & Sordet, Olivier \\
\hline Cerella, Claudia & Leo, Elisabetta & Sorrentino, Rosalinda \\
\hline Chen, Fei & Le-Rademacher, Jennifer & Sriuranpong, Virote \\
\hline Chen, Xiao & Li, Jianfeng & Stein, Ulrike \\
\hline Christopherson, Richard I & Li, Fengzhi & Sueta, Aiko \\
\hline Ciccolini, Joseph & Li, Wei & Syed, Nelofer \\
\hline Curtin, Nicola J. & Li, Xiujun (James) & Szlosarek, Peter W. \\
\hline Daidone, Maria Grazia & Lo Nigro, Cristiana & Tanaka, Yoichi \\
\hline Dandawate, Prasad & Mahadevan, Daruka & Terry, Stéphane \\
\hline Das, Benu Brata & Majello, Barbara & Tripathi, Manish K. \\
\hline De, Pradip & Manier, Salomon & Tsuda, Naotake \\
\hline De Lalla, Claudia & Marco, Giuseppetti Gian & Ueda, Takanori \\
\hline Dellabona, Paolo & Martin, Margarita & Urbatsch, Ina L. \\
\hline Dharmawardhane, Suranganie & Michaelis, Martin & Vallacchi, Viviana \\
\hline Domenicotti, Cinzia & Miller, John H. & Van Houten, Bennett E. \\
\hline Donnini, Sandra & Minderman, Hans & Van Roy, Nadine \\
\hline Duan, Bin & Mittal, Karuna & Van Tine, Brian A. \\
\hline Dudás, József & Monica, Silvia La & Van Waardenburg, Robert C.A.M. \\
\hline Elshimali, Yahya & Morales, Serafin & Venkatachalam, Annapoorna \\
\hline Essmann, Frank & Morbidelli, Lucia & Verdijk, Robert M. \\
\hline Falasca, Marco & Morgan, Thomas & Vergara, Daniele \\
\hline Falconi, Mattia & Morgenstern, Daniel A. & Viola, Giampietro \\
\hline Fan, Ping & Moro, Loredana & Viswakarma, Navin \\
\hline Fang, Qingming & Mostaghel, Elahe A. & Vitale, Giovanni \\
\hline Feun, Lynn G. & Mousa, Shaker & Viveiros, Miguel \\
\hline Fiandalo, Michael & Mraz, Marek & Wakimoto, Hiroaki \\
\hline Finlay, Graeme & Muzio, Giuliana & Wei, Yanzhang \\
\hline Gandhi, Varsha V. & Nakamura, Yuichi & Whittaker, Steven \\
\hline Gassman, Natalie R. & Neubauer, Hans & Wiemer, Erik A.C. \\
\hline Gaynon, Paul S & Neuman, Keir & Wilk, Anna \\
\hline Gemmill, Robert & Ocker, Matthias & Williams, Mark \\
\hline Ghosh, Chandrayee & Ogino, Shuji & Woolfenden, James M. \\
\hline Giampieri, Riccardo & Örfi, László & Wu, Lan-Xiang \\
\hline Giorgi, Ugo De & Pagano, Michele & Xu, Xiang-Xi \\
\hline Girotti, Albert & Panchapakesan, Balaji & Yague, Ernesto \\
\hline Gmeiner, William Henry & Park, Sungsu & Yahya, Elshimali \\
\hline Golubovskaya, Vita M. & Patil, Tejas & Yamauchi, Takahiro \\
\hline Grivicich, Ivana & Perry, Jo K. & Yang, Eddy S. \\
\hline Guo, Xiu-Li & Peterson, Tim & Yee, Douglas \\
\hline Hadizadeh, Farzin & Pillozzi, Serena & Yoon, Karina \\
\hline Hanemann, C. Oliver & Pizzimenti, Stefania & Yue, Wei \\
\hline Hempel, Georg & Pore, Milind & Zatelli, Maria Chiara \\
\hline Hnízda, Aleš & Pranay, Atul & Zheng, Shu-Sen \\
\hline Hochmair, Maximilian & Premkumar, Daniel & \\
\hline
\end{tabular}

We greatly appreciate the contribution they made and the time they donated to the journal. They can get their Reviewer Recognition Certificates by building their peer review profiles either on Publons (https:// publons.com) or on ORCID (https://orcid.org/).

If you are interested in becoming a reviewer for $C D R$, you are welcome to apply at https://cdrjournal.com/ journal/vol_reviewer. 


\section{DECLARATIONS}

Authors' contributions

Writing and revising the article and approving the final version: Cancer Drug Resistance Editorial Office.

Availability of data and materials

Not applicable.

Financial support and sponsorship

None.

\section{Conflicts of interest}

All authors declared that there are no conflicts of interest.

\section{Ethical approval and consent to participate}

Not applicable.

\section{Consent for publication}

Not applicable.

\section{Copyright}

(c) The Author(s) 2020. 